\title{
Diagnostic intervals before and after implementation of cancer patient pathways - a GP survey and registry based comparison of three cohorts of cancer patients
}

\author{
Henry Jensen ${ }^{1,2^{*}}$, Marie Louise Tørring ${ }^{1}$, Frede Olesen ${ }^{1}$, Jens Overgaard ${ }^{3}$, Morten Fenger-Grøn ${ }^{1}$ and Peter Vedsted ${ }^{1}$
}

\begin{abstract}
Background: From 2008, Danish general practitioners could refer patients suspected of having cancer to standardised cancer patient pathways (CPPS).

We aimed to compare the length of the diagnostic interval in 2010 with the length of the diagnostic interval before (2004/05) and during (2007/08) the implementation of CPPs in Denmark for all incident cancer patients who attended general practice prior to the cancer diagnosis.

Methods: General practitioner questionnaires and register data on 12,558 patients were used to compare adjusted diagnostic interval across time by quantile regression.

Results: The median diagnostic interval was $14(95 \% \mathrm{Cl}: 11 ; 16)$ days shorter during and $17(95 \% \mathrm{Cl}: 15 ; 19)$ days shorter after the implementation of CPPs than before. The diagnostic interval was 15 (95\% Cl: 12;17) days shorter for patients referred to a CPP in 2010 than during the implementation, whereas patients not referred to a CPP in 2010 had a 4 ( $95 \%$ Cl: $1 ; 7$ ) days longer median diagnostic interval; the pattern was similar, but larger at the $75^{\text {th }}$ and $90^{\text {th }}$ percentiles.

Conclusion: The diagnostic interval was significantly lower after CPP implementation. Yet, patients not referred to a CPP in 2010 tended to have a longer diagnostic interval compared to during the implementation. CPPs may thus only seem to expedite the diagnostic process for some cancer patients.
\end{abstract}

Keywords: Diagnostic interval, Urgent referral, (early) diagnosis, Cancer, Primary care, Cohort, Denmark

\section{Background}

Standardised cancer patient pathways (CPPs) have been introduced in some countries [1-8]. Even though CPP contents differ between countries, they all operate with a guaranteed timeframe for timely diagnosis. After years of increasing waiting times for cancer patients in Denmark, the Danish government and the Danish regions (i.e. hospital owners) declared in 2007 that cancer should be diagnosed and treated without delay [9]. Consequently, the Danish government and the Danish National Board

\footnotetext{
* Correspondence: henry.jensen@feap.dk

${ }^{1}$ Research Centre for Cancer Diagnosis in Primary Care, Research Unit for General Practice, Department of Public Health, Aarhus University, Bartholins Allé 2, DK-8000 Aarhus C, Denmark

${ }^{2}$ Section for General Medical Practice, Department of Public Health, Aarhus University, Bartholins Allé 2, DK-8000 Aarhus C, Denmark

Full list of author information is available at the end of the article
}

of Health (today: the Danish Health and Medicines Authority) introduced CPPs in Denmark in 2008 [2]. CPPs were introduced in Denmark under the assumption that timely diagnosis and decisions on treatment options could be enhanced, psychosocial distress limited, and ultimately improve the prognosis for cancer patients. The Danish CPPs are standardised pathways for the time up to the final diagnosis and the start of treatment comprising well-defined sequences and time frames for diagnostic procedures and treatments for patients fulfilling CPP access criteria. The Danish CPPs were accompanied by renewal and expansion of equipment for imaging and radiotherapy. Patients can be referred to a Danish CPP when the clinician has a reasonable suspicion of cancer as the final diagnosis [2]. 
A key phase in the cancer journey is the diagnostic interval (DI), i.e. the time from the patient's first presentation of symptoms in the health care system (usually in primary care) until diagnosis [10]. Despite a sparse body of evidence [11], mortality has been shown to increase with longer DI among patients with colorectal, lung, breast, melanoma or prostate cancers [12]. The DI is important as it measures the timeliness of the health-care system as a whole across sector boundaries.

Few studies have addressed the possible CPP impact on DI length. Most of these studies have primarily focussed on selected parts of the DI for specific cancer types, or studies have been performed with no baseline measures [1,4-7]. In addition, some studies are restricted to include only patients with predefined symptoms of cancer [13] or exclusively patients referred for a CPP [6] although some studies have reported that fairly few cancer patients initially present with well-known symptoms of cancer that allow direct access to a CPP $[14,15]$. Furthermore, many of the patients who are not referred to diagnostic workup through a CPP experience longer time intervals [7,16-18]. Thus, the possible effect of CPP implementation might vary with the actual use of CPPs, patient symptomatology and cancer types.

Therefore, we aimed to compare the length of the diagnostic interval in 2010 with the length of the diagnostic interval before (2004/05) and during (2007/08) the implementation of CPPs in Denmark for all incident cancer patients who attended general practice prior to the cancer diagnosis and for the five most common cancer types, regardless of the patients' presenting symptoms.

\section{Methods}

Data from GPs and registries from the Danish Cancer in Primary Care (CaP) cohort [19] was used in an ecological design to compare three cohorts of incident cancer patients before, during and after CPP implementation in order to investigate the impact of the CPP implementation in 2007-2009 [2] as a natural experiment. The ecological design was a consequence of the unknown joint distribution of CPP referral prior to the CPP implementation [20].

\section{Setting}

The study took place in Denmark, where the annual cancer incidence rate is 326 per 100,000 [21]. The Danish publicly funded healthcare system ensures a uniform healthcare system with free access to diagnostics and treatment for all citizens. More than $98 \%$ of all citizens are registered with a specific general practice, which they may consult for medical advice. The Danish general practitioners (GPs) act as gatekeepers to the rest of the health care system. During the study period, $78.6 \%$ of all cancer patients in Denmark had been diagnosed through a primary care route [19].

\section{Patient population and data collection}

Identification of patients, data collection and response analysis were based on the large Cancer in Primary Care study, which has been described in detail elsewhere [19]. In brief, we identified incident cancer patients aged 18 years or above and listed with a Danish GP and for whom diagnoses were coded according to the International Classification of Diseases, version 10 (ICD-10), i.e. C00.0-C99.9 (except for non-melanoma skin cancer (C44)), before from the former Danish County of Aarhus (640,000 inhabitants) (1 September 2004 - 31 August 2005), during from the Region of Southern Denmark and the Central Denmark Region (2.1 million inhabitants) (1 October 2007 - 30 September 2008) and after, from entire Denmark (1 May - 31 August 2010), the implementation of the CPPs at national level. Patients were identified through the Patient Administrative Systems (PAS) of the Danish hospitals and the Danish National Patient Registry. A patient was defined to have incident cancer when cancer was registered as the primary diagnosis during one of the inclusion periods, and no prior history of cancer was recorded. The history of cancer was checked in the Danish Cancer Registry [22]. We excluded 570 out of 22,736 patients (3\%) because their diagnosis could not be verified by the Danish Cancer Registry.

For each sampled cancer patient, data from the registered GP was collected by a questionnaire, which was sent to the GP 2-5 weeks after identification of the patient. Participating GPs were asked to fill in the questionnaire on the basis of the information in their electronic medical records. Non-responders received a reminder after 3-5 weeks. Information from the questionnaire was combined with data from the Danish Cancer Registry to ensure that we obtained a validated date of diagnosis [22].

Figure 1 shows the patient flow in the present study. In $4,603(20.8 \%)$ of the 22,169 verified cases, the invited GPs did not respond (GP response rate: 79.2\%). Patients with responding GPs did not differ from patients with non-responding GPs in regard to 1-year survival, comorbidity or educational level. However, patients listed with responding GPs were more likely to be women, younger, to have a higher disposable income, to have more regionally or distantly spread tumours, and correspondingly more likely to have breast cancer and less likely to have prostate cancer than patients from non-responding GPs. These differences were small and clinically irrelevant, but were statistically significant due to the sample size (data published elsewhere) [19]. We excluded 3,766 (21.4\%) patients from this study as the GP stated that (s)he was not involved in the diagnosis; i.e. patients diagnosed through screening, emergency access or as coincidental findings during diagnosis of other illnesses. We also excluded 15 male breast cancer patients (0.1\%) (Figure 1 ). 


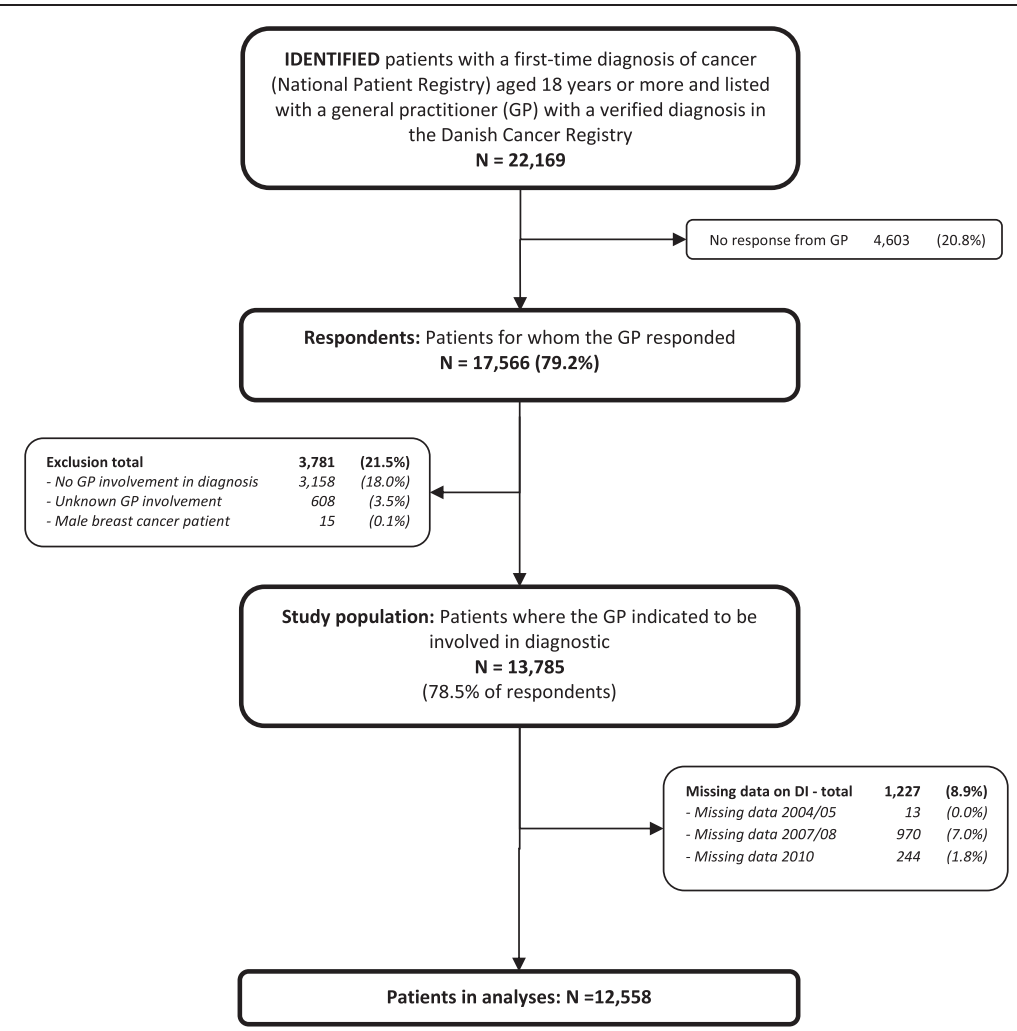

Figure 1 Flowchart for cancer patients. Boxes on the left indicate exclusion of patients who did not meet the inclusion criteria and boxes on the right indicate drop-outs due to non-response and missing data.

\section{Outcome, exposure and possible confounders}

In accordance with the Aarhus Statement, we defined the primary outcome, the DI, as the time from when the patient made the first symptom presentation to a GP until the time of diagnosis [10]. The DI was calculated by using the GP questionnaire to obtain the date of the patient's first presentation of symptoms to the GP and the Danish Cancer Registry to define the date of diagnosis. The date of diagnosis recorded in this registry corresponds to the date of first contact (admission date) with the hospital department at which the cancer diagnosis was first registered as the primary cause of contact. If the patient was diagnosed by a private practicing specialist, the date of diagnosis corresponds to the date of the clinical diagnosis [23]. If the date of diagnosis was missing in Danish Cancer Registry, the admission date recorded in the Danish National Patient Registry was used.

Exposure was defined as implementation of CPP, and each of the three sub-cohorts was treated as an independent exposure group: 2004/05 = no CPPs implemented (before), 2007/08 = CPPs under implementation (during) and 2010 = fully implemented CPPs (after). Subsequently, we subdivided the 'after' group into two groups: patients who were initially referred to a CPP (after-CPP) and patients who were not (after-no CPP).
Possible confounders accounted for were gender, age, comorbidity, educational level and disposable income. Gender and age were derived from the patient's Danish civil registration number [24]. We computed a Charlson Comorbidity Index score according to the method described by Quan et al. [25] using the date of the patient's first consultation with the GP as the index date. We grouped the comorbidity scores into 'none' (no recorded disease), 'moderate' (score of 1 or 2), and 'high' (score of 3 or more). We grouped educational levels according to the International Standard Classification of Education (ISCED) [26] into 'low' (ISCED levels 1 and 2), 'medium' (ISCED levels 3 and 4) and 'high' (ISCED levels 5 and 6), and we categorized disposable household OECD income in the year prior to the diagnosis into tertiles ('low', 'medium' and 'high').

\section{Ethics}

The study was approved by the Danish Data Protection Agency (file. no. 2009-41-3471). The Danish National Board of Health gave legal permission to obtain information from the GPs' medical records. The study did not require approval from the Committee on Health Research Ethics of the Central Denmark Region as no biomedical intervention was performed. 


\section{Analysis}

We analysed changes in the DI for all cancers combined and for each of the five most common cancer types in Denmark: colorectal, lung, malignant melanoma, breast and prostate cancer [27].

All statistical analyses, except for analyses of missing data and sensitivity, were restricted to complete cases (i.e. GPs who completed the questionnaire and who were also involved in the diagnostic process).

Prefatory comparisons of the three sub-cohorts were performed using non-parametric methods: The Chi2 test was used for categorical data, while the Wilcoxon's rank sum test was used for continuous data.

We used the qcount procedure written by Miranda [28] for the quantile regression analyses [29] on the smoothed quantiles to estimate the adjusted differences in the diagnostic interval at different percentiles; analysis on the smoothed quantiles are recommended for analyses of discrete (count) data [30]. Two adjusted models were considered: a model with no regard of referral route (overall trend) and a model with patients after the CPP implementation in 2010 divided into referral routes (trend by referral route). We adjusted for gender, age, cancer site, comorbidity, educational level and disposable income in both models. Age was centred at 45 years of age and was entered into the models as a continuous variable, while the other known confounders were entered as categorical variables.

To investigate the implication of missing data of DI, we performed best/worst case scenario sensitivity analyses by assigning the value 0 (best case) and the maximum values for the sub-cohorts (worst case) of the diagnostic intervals.

A statistical level of $\mathrm{p} \leq 0.05$ was considered significant in all analyses. Analyses were done using Stata ${ }^{\circ}$ statistical software, version 13 (StataCorp LP, College Station, TX, USA).

\section{Results}

\section{Demographic characteristics of excluded and included} patients

In total 13,785 patients fulfilled the inclusion criteria. We excluded 1,227 patients (8.9\%) due to missing information of the DI. These patients were more likely to be women, younger than 45 years of age or older than 75 years of age, to be diagnosed with breast or prostate cancers, to have high income or to have higher survival rates than the included patients. The characteristics of the analysed 12,558 are presented in Table 1.

\section{Diagnostic interval - overall tendency}

The unadjusted diagnostic intervals before, during and after CPP implementation are summarised in Table 2 and Figure 2. The median DI was statistically significantly lower across time: 49 (interquartile interval (IQI): 24;96) days before, 35 (IQI: 16;78) days during and 32 (IQI: 14;73) days after CPP implementation (Table 2 \& Figure 2). The overall result remained the same when we adjusted for differences between populations; the median DI was $14(95 \%$ CI: $11 ; 16)$ days shorter during the transition stage than before CPP implementation and 17 (95\% CI: 15;19) days shorter after CPP implementation (Table 3). Compared to the period before CPPs, the DI was shorter both during and after CPP implementation for all cancer types, although not statistically significant at all percentiles (Additional file 1).

\section{Diagnostic interval by referral route compared to before CPP implementation}

When patients diagnosed after CPP implementation were categorised according to the GPs use of CPP $62.8 \%$ were categorised as non-CPP referrals. The unadjusted median DI was lower for both the after-CPP group and the after-no CPP group compared to before the implementation of CPPs $(\mathrm{p}<0.001)$ (Table 2$)$. The DI was significantly longer for the after-no CPP group than for the after-CPP group ( $\mathrm{p}<0.001)$. This was observed for all the five major cancer types (Table 2 ). The $75^{\text {th }}$ percentile was 91 days in 2010 for the after-no CPP group compared to 44 days for the after-CPP group.

For the after-CPP group, the adjusted median was 23 (95\% CI: $21 ; 25$ ) days shorter than before the implementation. For the after-no CPP group, the adjusted median was 9 (95\% CI: 7;12) days shorter than before the implementation. At the $90^{\text {th }}$ percentile, the DI for the after-CPP group was 110 (95\% CI: 67;153) days shorter than before, while similar (6 (95\% CI: $-66 ; 77)$ days shorter) for the after-no CPP group than before (Table 3). This tendency was observed for all five major cancer types, although not statistically significant at all percentiles (Additional file 1).

\section{Diagnostic interval by referral route compared to during CPP implementation}

For the after-CPP group, the adjusted median DI was 15 (95\% CI: 12;17) days shorter than during the implementation. For the after-no CPP group, the adjusted median DI was 4 (95\% CI: 1;7) days longer than during the implementation. Likewise, at the $90^{\text {th }}$ percentile, the DI for the after-CPP group was 80 (95\% CI: 34;126) days shorter than during the implementation, while the DI for the after-no CPP group was insignificantly $48(95 \% \mathrm{CI}:-49 ; 145)$ days longer than during the implementation (Table 3). This tendency was observed for all cancer types separately, although not statistically significant at all percentiles (Additional file 1). 
Table 1 Patient characteristics displayed by cohort time and total $(\mathrm{N}=12,558)$

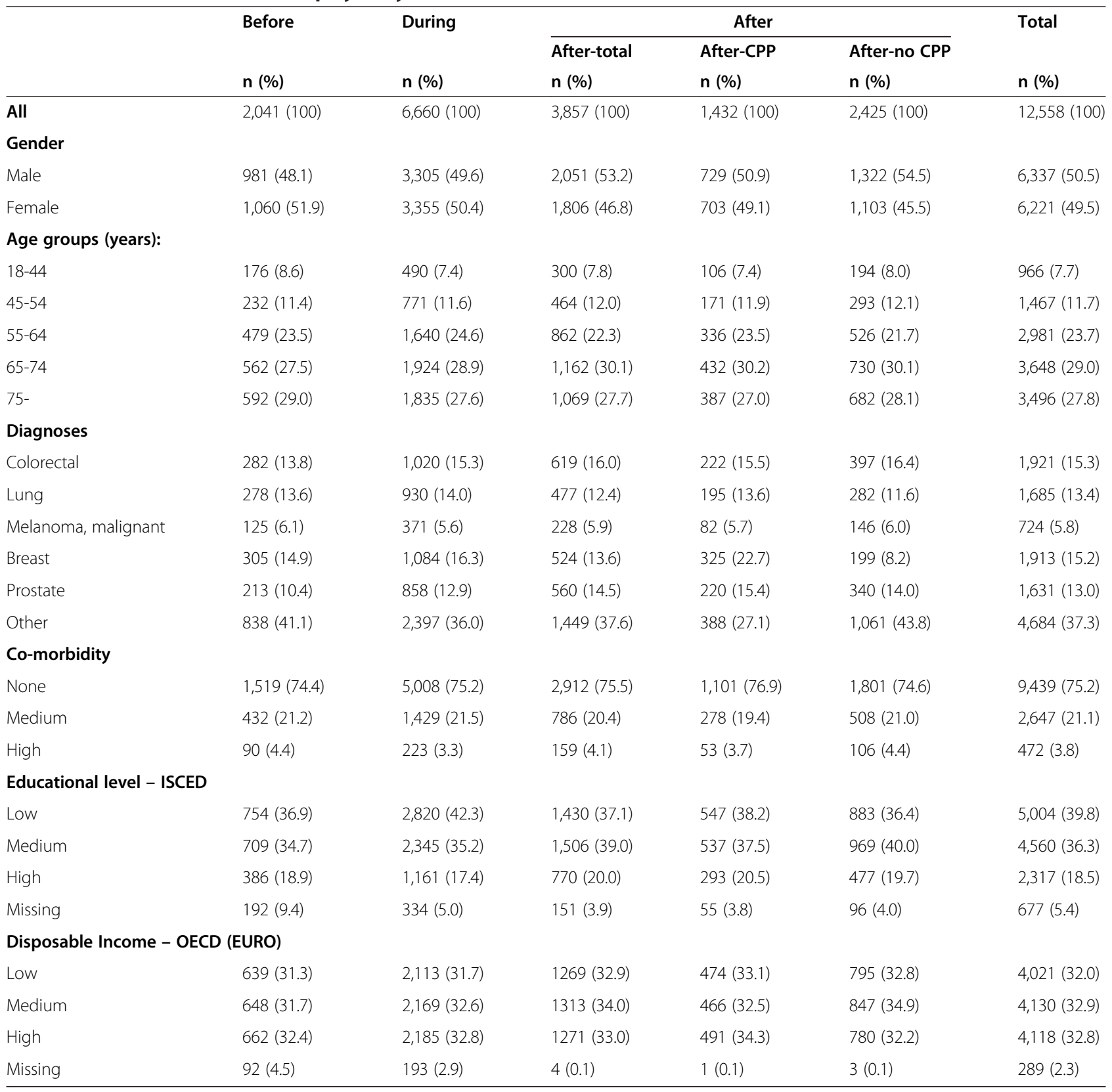

Column five and six display the 'after cohort' divided by referral route (referred to a Cancer Patient Pathway (after-CPP) or not (after-no CPP)).

\section{Sensitivity analysis}

The sensitivity analyses did not alter the overall results as the median DI was still lower both during and after the implementation compared to before; this was found for both worst and best case scenario. Furthermore, both scenarios displayed that the median DI was longer for the after-no CPP group than for the after-CPP and also showed that the after-no CPP group tended to experience longer DIs after than during CPP implementation. Sensitivity analyses restricted to the same geographical region showed similar results.

\section{Discussion}

We found that the median length of the DI in Denmark was shorter after the CPP implementation (in 2010) than before the CPP implementation (in 2004/05); the largest difference was found between the period before the CPPs (2004/05) and during the implementation phase in $2007 / 08$. Furthermore, the largest difference in DI (compared to before the implementation) was found among patients in the after-CPP group, whereas patients in the after-no CPP group had only a minimally lower DI (compared to before the implementation) and still a long DI. In fact, the after-no CPP group in 2010 displayed a 
Table 2 The unadjusted diagnostic interval (DI) shown before (2004/05), during (2007/08) and after (2010 - combined) the implementation of CPPs $(\mathrm{N}=12,558)$

\begin{tabular}{|c|c|c|c|c|c|}
\hline & \multirow[t]{2}{*}{ Before } & \multirow[t]{2}{*}{ During } & \multicolumn{3}{|c|}{ After } \\
\hline & & & After-total & After-CPP & After-no CPP \\
\hline & Median (IQI) & Median (IQI) & Median (IQI) & Median (IQI) & Median (IQI) \\
\hline Total & $49(24 ; 96)$ & $35(16 ; 78)$ & $32(14 ; 73)$ & $22(9 ; 44)$ & $41(17 ; 91)$ \\
\hline \multicolumn{6}{|l|}{ Gender } \\
\hline Female & $41(21 ; 83)$ & $31(14 ; 67)$ & $29(12 ; 63)$ & $18(7 ; 35)$ & $39(17 ; 80)$ \\
\hline Male & $56(28 ; 118)$ & $40(19 ; 89)$ & $35(14 ; 82)$ & $26(12 ; 54)$ & $43(18 ; 102)$ \\
\hline \multicolumn{6}{|l|}{ Age groups } \\
\hline $18-44$ years & $42(21 ; 93)$ & $32(15 ; 69)$ & $30(13 ; 72)$ & $16(8 ; 29)$ & $49(21 ; 92)$ \\
\hline $45-54$ years & $46(22 ; 84)$ & $31(15 ; 64)$ & $27(13 ; 55)$ & $21(8 ; 34)$ & $34(16 ; 64)$ \\
\hline $55-64$ years & $46(23 ; 87)$ & $31(15 ; 71)$ & $30(13 ; 64)$ & $20(8 ; 41)$ & $39(18 ; 80)$ \\
\hline $65-74$ years & $49(25 ; 98)$ & $38(18 ; 82)$ & $35(15 ; 78)$ & $26(12 ; 53)$ & $42(17 ; 95)$ \\
\hline 75 years and above & $55(26 ; 107)$ & $38(16 ; 91)$ & $34(13 ; 84)$ & $22(8 ; 53)$ & $45(17 ; 109)$ \\
\hline \multicolumn{6}{|l|}{ Diagnoses } \\
\hline Colorectal & $55(27 ; 95)$ & $37(17 ; 84)$ & $31(14 ; 69)$ & $22(8 ; 46)$ & $37(18 ; 80)$ \\
\hline Lung & $49(27 ; 85)$ & $28(12 ; 64)$ & $28(11 ; 67)$ & $21(9 ; 47)$ & $33(12 ; 80)$ \\
\hline Melanoma, malignant & $39(23 ; 67)$ & $35(17 ; 69)$ & $28(12 ; 55)$ & $15(6 ; 29)$ & $37(19 ; 74)$ \\
\hline Breast & $36(19 ; 56)$ & $21(13 ; 36)$ & $18(8 ; 34)$ & $13(5 ; 23)$ & $29(15 ; 51)$ \\
\hline Prostate & $85(44 ; 160)$ & $57(30 ; 128)$ & $46(21 ; 108)$ & $34(19 ; 75)$ & $53(23 ; 168)$ \\
\hline Other & $51(22 ; 113)$ & $42(18 ; 95)$ & $40(16 ; 88)$ & $29(11 ; 56)$ & $48(18 ; 100)$ \\
\hline \multicolumn{6}{|l|}{ Co-morbidity } \\
\hline None & $49(23 ; 93)$ & $34(16 ; 77)$ & $31(14 ; 71)$ & $21(9 ; 42)$ & $41(18 ; 91)$ \\
\hline Medium & $49(27 ; 106)$ & $38(16 ; 83)$ & $34(14 ; 78)$ & $26(8 ; 53)$ & $41(16 ; 92)$ \\
\hline High & $43(14 ; 88)$ & $35(14 ; 78)$ & $31(13 ; 78)$ & $19(6 ; 47)$ & $46(17 ; 93)$ \\
\hline \multicolumn{6}{|c|}{ Disposable income - OECD } \\
\hline Low & $50(22 ; 106)$ & $36(16 ; 83)$ & $32(13 ; 77)$ & $22(8 ; 50)$ & $42(16 ; 92)$ \\
\hline Medium & $49(24 ; 95)$ & $35(16 ; 77)$ & $32(14 ; 77)$ & $22(9 ; 43)$ & $41(18 ; 92)$ \\
\hline High & $50(27 ; 93)$ & $34(16 ; 76)$ & $31(14 ; 68)$ & $22(9 ; 41)$ & $41(19 ; 85)$ \\
\hline Missing & $32(12 ; 67)$ & $34(15 ; 68)$ & $50(24 ; 66)$ & $3(3 ; 3)$ & $55(45 ; 77)$ \\
\hline \multicolumn{6}{|c|}{ Educational level - ISCED } \\
\hline Low & $49(22 ; 98)$ & $35(16 ; 79)$ & $32(14 ; 73)$ & $23(9 ; 49)$ & $42(17 ; 89)$ \\
\hline Medium & $47(25 ; 95)$ & $34(16 ; 76)$ & $32(14 ; 75)$ & $21(9 ; 43)$ & $40(18 ; 91)$ \\
\hline High & $51(28 ; 97)$ & $36(18 ; 83)$ & $31(14 ; 71)$ & $22(8 ; 41)$ & $42(18 ; 92)$ \\
\hline Missing & $49(21 ; 92)$ & $29(11 ; 73)$ & $32(10 ; 71)$ & $24(5 ; 40)$ & $40(14 ; 92)$ \\
\hline
\end{tabular}

Column five and six display the 'after cohort' divided by referral route (referred to a Cancer Patient Pathway (after-CPP) or not (after-no CPP)).

longer median DI than during the implementation. Finally, we found that the $90^{\text {th }}$ percentile DI for the after-no CPP group did not differ from before the CPP implementation. Hence, only patients in the CPP group in 2010 had a lower diagnostic interval after the CPP implementation than before CPP.

This finding must be compared to the fact that $63 \%$ of all Danish cancer patients in 2010 were not initially referred to a CPP [18]. Hence, the majority of cancer patients did not experience a lower DI across the investigated time period. This may have major impact on the prognosis for both patients with long DIs and at a population level, as it is reasonable to assume that expedited diagnosis of symptomatic cancer is likely to benefit the patients in terms of improved survival [12,31-35]. Hence, reductions in the diagnostic intervals (as we have shown) may influence the cancer stage distribution and hence survival at population level; these relations have been claimed to partly explain the improvement in survival among Danish cancer patients [36,37]. However, there is not yet enough evidence to substantiate this argument. Another equally important 


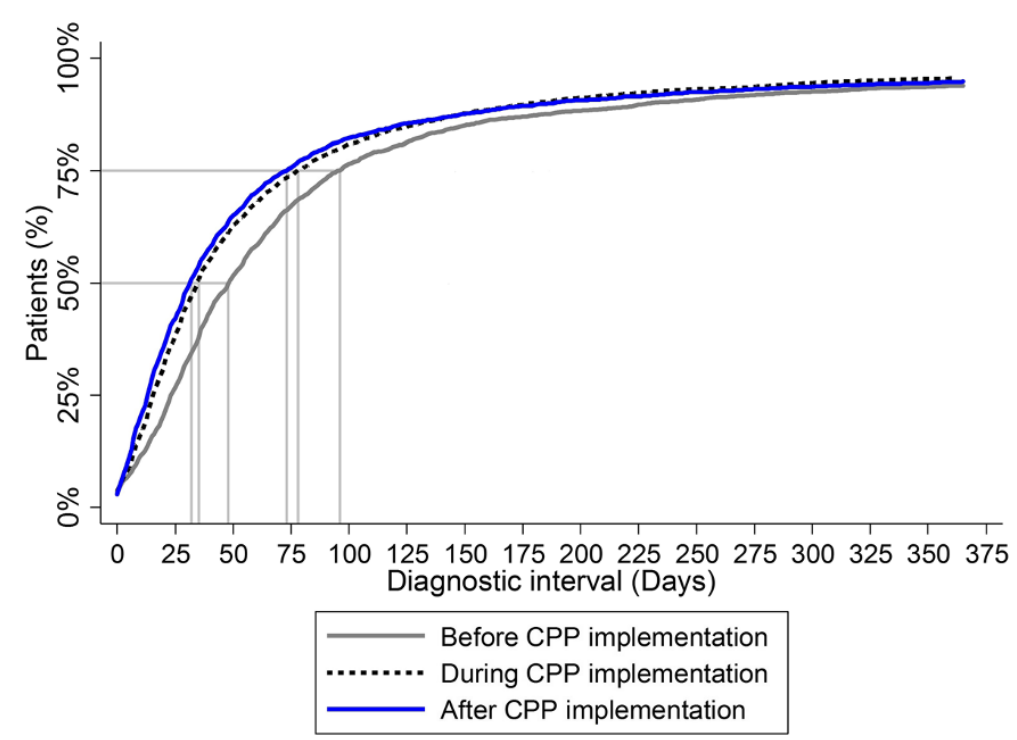

Figure 2 Cumulative frequencies of diagnostic interval (DI) before, during and after CPP implementation in Denmark. DI ranked in order and depicted by a Lorenz diagram. DI of longer than 365 days omitted for illustration purposes.

effect of reducing the diagnostic interval is that it should improve patient satisfaction and limit psychological distress among cancer patients, which was another important aim of the CPPS [2].

The literature on DI is sparse and direct comparisons between studies and countries are difficult [10]. We know of only two studies that have investigated the DI across time periods in connection with implementation of CPPs: a Danish study on head and neck cancer [5] and the large UK study on the implementation of the NICE guidelines [13]. Our results, which show a shorter DI after CPP implementation, are in line with the shorter DI found by these two studies. Yet, our study is the first to quantify adjusted changes in the DI across time at different percentiles. Our findings display that the decrease in the DI across time was largest among the patients who waited the longest, which may have major impact on the prognosis. Furthermore, we were able to quantify the changes in the DI across time by use of CPPs. We found that the patients referred to a CPP have a significantly shorter DI than patients not referred to a CPP at all percentiles. These findings are in line with the results of our previous studies in which we also accounted for the patients' symptom presentation [18].

The design of the study does not permit us to infer causality between the implementation of CPPs and the lower DI seen across time. A number of changes in policy, clinical practice and investments may have

Table 3 Estimated differences in diagnostic interval (DI) (calendar days) during and after the implementation of CPPs compared to before the implementation (Model 1), and also according to referral route after the implementation: to a CPP (after-CPP) or not (after-no CPP) (Model 2) (N=11,640)

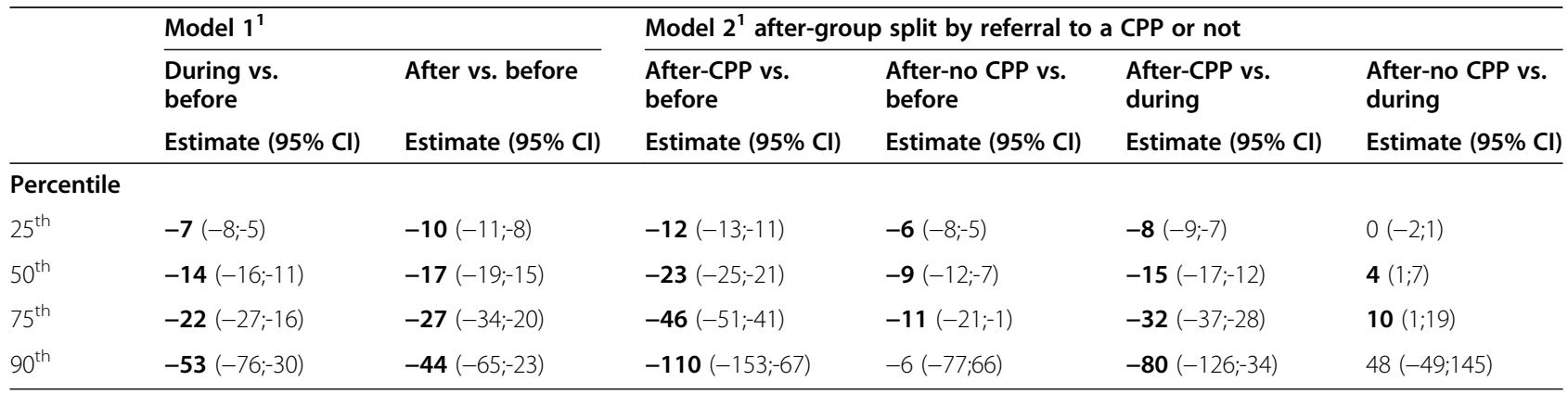

Estimates with $95 \%$ confidence intervals $(95 \% \mathrm{Cl})$ are displayed for the $25^{\text {th }}$, the $50^{\text {th }}$, the $75^{\text {th }}$ percentile and the $90^{\text {th }}$ percentiles. Bold estimates indicate statistical significance at $\mathrm{p}=0.05$ level or less.

Model 1 reference: before implementation group, cohort, female, 45 years of age, cancer sites, no co-morbidity, high disposable income and high educational level.

Model $2=$ model 1 , but with 'after group' split by referral route (CPP)

${ }^{1}$ Adjusted for gender, age, cancer site, co-morbidity, educational level and disposable income. 
contributed to these changes in DI across time. The implementation of CPPs was just one of many new initiatives in the second Danish cancer plan, which also promoted a huge expansion in radiotherapy facilities [38]. As the largest difference in DI was observed from before to during the implementation of CPPs, most of the differences found can probably not be attributed to the (full) implementation of the CPPs. The decision to implement CPPs was taken in August 2007, just after a declaration by the prime minister and the Danish National Board of Health that cancer should be treated without any delay in Denmark [9]. Therefore, local leaders may have started to streamline the diagnostic trajectories already before the official implementation in 2008/09, which could have contributed to the lower DIs observed in $2007 / 2008$. Our study further shows that the general tendency towards lower diagnostic interval after the full implementation of the CPPs was different between patient referred to a CPP and patients who were not.

\section{Clinical implications}

The results found in the study-together with other findings of longer intervals among patients not referred to rapid diagnostics $[16,17]$ - supports the argument that introduction of CPPs only benefit patients referred to a CPP [17]. This has been suggested to be due to that the 'fast-track' system may disadvantage the large group of patients in whom the first appearance of disease does not involve significant cardinal symptoms of cancer $[39,40]$. This is underlined by the different proportion of CPP referrals between cancer types with e.g. breast cancer most often referred to a CPP [18]. Our findings may thus be interpreted as a demonstration of the possible danger of considering standardised CPPs as stand-alone referral routes for cancer. Our results may also indicate a need for an additional approach to ensure fast diagnosis of cancer, for instance by providing quick and easy access from primary care to all initial investigations ordered by a GP to establish the possibility of cancer [3].

\section{Strengths and weaknesses of the study}

The main strength of this study is the study population, which was well-defined and complete with minimal selection bias $[19,41]$. We may have missed some patients, but this risk is expected to be negligible as we also included late-registered patients [41]. Another major strength that decreases the risk for selection bias is that we included all cancer patients, regardless of symptom presented at first contact and cancer site.

The Danish health care system is almost uniformly organized across different geographically and administratively independent regions. This organization allowed the merging of the three sub-cohorts into one, although they originated from partly overlapping geographical locations (regions) in Denmark and thus belonged to different subsets. In fact, the case-mix of the sub-cohorts resembles the case-mix in the DCR of a given year $[41,42]$. This indicates that the identified incident patients in the $\mathrm{CaP}$ Cohort are representative of incident cancer patients in Denmark at the time when the patients were identified.

The considerable size of the study ensures statistical precision, and the high response rate of $79 \%$ reduces the risk of selection bias. However, patients who were not included may have had longer DIs than the included patients. We believe this is not associated with the implementation of CPPs and, therefore, would not bias the observed DI differences between the sub-cohorts. Nevertheless, selection bias may still be present, but as our sensitivity analyses showed no impact on the results, this possible bias has been considered to be negligible (if present at all).

Information bias due to GP recall bias was reduced by using the GPs' contemporaneously updated electronic medical records. Even so, the retrospective nature of the questionnaire holds the risk that the GPs may have misinterpreted the date of first presentation of symptoms for some of the cases. We believe that this possible risk is equal for all sub-cohorts and consequently will not bias the DI differences between the cohorts.

For obvious reasons, it is not possible to identify the patients in the before and during cohort, who would have been referred to CPPs had they been implemented. It is likely, that patients not eligible for CPPs would have had a tendency to longer DIs before and during the implementation. Hence, the fact that patients not referred to CPP have longer DI than the 'during' cohort as a whole, cannot be rigorously interpreted as a causal effect of CPPs disadvantaging this group. Furthermore, as the categorisation of patients according to CPP or not was based on the GPs choice of referral, comparison with all patients diagnosed at hospitals using CPPs must be cautioned.

The use of the date of first contact to a hospital ward as the date of diagnosis would tend to underestimate the length of the DI. This standard procedure is caused by the Danish Cancer Registry as the first contact date is recorded in this register as the date of diagnosis, even though most diagnoses are verified after this date (mostly at a multidisciplinary team meeting at the hospital). We consider this to be non-differential as we suspect that it is not associated with the CPP implementation, and hence deviations in date of diagnosis alone cannot explain the observed differences in DI between the cohorts. Yet, if this information bias may have been stronger for patients not referred to a CPP (after-no CPP group) as these patients have longer intervals and thereby may have raised the possibility that the date of diagnosis was moved relatively more than for the other groups of 
patients, this bias could have led to an underestimation of the observed DI difference between the after-no CPP group and the other groups. Our observed differences would thus be minimum estimates of the true differences.

\section{Conclusion}

The diagnostic interval for the five most common cancers and for all cancers combined was lower in Denmark in 2010 than in 2004/05. The largest difference was seen from 2004/05 to 2007/08. Patients who were not referred to a CPP in 2010 still had long diagnostic intervals and tended to have a longer diagnostic interval than patients in 2007/08 when the CPP was not fully implemented. The patients with the $10 \%$ longest waiting time and who were not referred to a CPP in 2010 actually displayed a DI similar to the DI for the $10 \%$ waiting the longest in 2004/05.

These findings suggest that, despite the good intentions with implementing the CPPs, patients who were not referred to a CPP seem not to have gained faster diagnosis as these patients tend to have similar diagnostic intervals as before the implementation of CPPs. This demonstrates a need for more focus on providing faster diagnostic pathways for the large groups of patients who are not referred to a CPP in the initial phases of their disease.

\section{Additional file}

Additional file 1: Estimated differences in diagnostic intervals (DIs) after and during CPP implementation compared to before, by cancer type.

\section{Abbreviations}

CPP: Standardised Cancer Patient Pathways; GP: General practitioner; Cl: 95\% Confidence Interval; CCI: Charlson Co-morbidty Index; ISCED: International Standard Classification of Education.

\section{Competing interests}

The authors declare to have no competing interests.

\section{Authors' contributions}

HJ was involved in the conception of the study, participated in the study's design, performed the statistical analyses and drafted the manuscript. $\mathrm{MLT}, \mathrm{FO}, \mathrm{JO}$ and PV all contributed to the conception, development and design of the study and provided critical revision of the intellectual contents of the manuscript. MFG contributed to the conception of the study and the statistical analysis and provided critical revision of the intellectual contents of the manuscript. All authors have read and approved the final manuscript.

\section{Acknowledgements}

We would like to thank data manager Kaare Rud Flarup for his outstanding and meticulous help in setting up and maintaining the database and enabling register linkage at Statistics Denmark. We thank Statistics Denmark for providing the data platform and the secure data environment.

\section{Research support}

This study was funded by the Novo Nordisk Foundation, the Danish Cancer Society, the Health Foundation (Helsefonden), the Danish foundation Trygfonden and the Central Denmark Region Foundation for Primary Health Care Research (Praksisforskningsfonden).

\section{Author details}

'Research Centre for Cancer Diagnosis in Primary Care, Research Unit for General Practice, Department of Public Health, Aarhus University, Bartholins Allé 2, DK-8000 Aarhus C, Denmark. ${ }^{2}$ Section for General Medical Practice, Department of Public Health, Aarhus University, Bartholins Allé 2, DK-8000 Aarhus C, Denmark. ${ }^{3}$ Department of Clinical Medicine - Department of Experimental Clinical Oncology, Aarhus University Hospital, Noerrebrogade 44, DK-8000 Aarhus C, Denmark.

Received: 11 December 2014 Accepted: 16 April 2015

Published online: 23 April 2015

\section{References}

1. Prades J, Espinas JA, Font R, Argimon JM, Borras JM. Implementing a Cancer Fast-track Programme between primary and specialised care in Catalonia (Spain): a mixed methods study. Br J Cancer. 2011;105:753-9.

2. Probst $H B$, Hussain $Z B$, Andersen O. Cancer patient pathways in Denmark as a joint effort between bureaucrats, health professionals and politicians-A national Danish project. Health Policy. 2012;105:65-70.

3. Olesen F, Hansen RP, Vedsted P. Delay in diagnosis: the experience in Denmark. Br J Cancer. 2009;101 Suppl 2:S5-8.

4. Toustrup K, Lambertsen K, Birke-Sorensen H, Ulhoi B, Sorensen L, Grau C Reduction in waiting time for diagnosis and treatment of head and neck cancer - a fast track study. Acta Oncol. 2011;50:636-41.

5. Lyhne NM, Christensen A, Alanin MC, Bruun MT, Jung TH, Bruhn MA, et al. Waiting times for diagnosis and treatment of head and neck cancer in Denmark in 2010 compared to 1992 and 2002. Eur J Cancer. 2013:49:1627-33.

6. Vallverdu-Cartie H, Comajuncosas-Camp J, Orbeal-Saenz RA, Lopez-Negre JL, Gris Garriga PJ, Jimeno-Fraile J, et al. Results of implementation of a fast track pathway for diagnosis of colorectal cancer. Rev Esp Enferm Dig. 2011;103:402-7.

7. Valentin-Lopez B, Ferrandiz-Santos J, Blasco-Amaro JA, Morillas-Sainz JD, Ruiz-Lopez P. Assessment of a rapid referral pathway for suspected colorectal cancer in Madrid. Fam Pract. 2012;29:182-8.

8. Department of Health. The NHS Cancer Plan. A plan for investment, A plan for reform. London: Department of Health; 2000.

9. Danish National Board of Health: Aftale om gennemførelse af målsætningen om akut handling og klar besked til kræftpatienter [Agreement on acute action and clear information to cancer patients]; [https://sundhedsstyrelsen.dk/ /media/ A92B68A03752493FA62EA08BA169FC05.ashx] (Accessed 16-03-2015).

10. Weller D, Vedsted P, Rubin G, Walter FM, Emery J, Scott S, et al. The Aarhus statement: improving design and reporting of studies on early cancer diagnosis. Br J Cancer. 2012;106:1262-7.

11. Neal RD. Do diagnostic delays in cancer matter? Br J Cancer. 2009;101 Suppl 2:S9-12.

12. Torring ML, Frydenberg M, Hansen RP, Olesen F, Vedsted P. Evidence of increasing mortality with longer diagnostic intervals for five common cancers: a cohort study in primary care. Eur J Cancer. 2013;49:2187-98.

13. Neal RD, Din NU, Hamilton W, Ukoumunne OC, Carter B, Stapley S, et al, Comparison of cancer diagnostic intervals before and after implementation of NICE guidelines: analysis of data from the UK General Practice Research Database. Br J Cancer. 2014;110:584-92.

14. Scheel BI, Ingebrigtsen SG, Thorsen T, Holtedahl K. Cancer suspicion in general practice: the role of symptoms and patient characteristics, and their association with subsequent cancer. Br I Gen Pract. 2013;63:627-35.

15. Ingebrigtsen SG, Scheel BI, Hart B, Thorsen T, Holtedahl K. Frequency of 'warning signs of cancer' in Norwegian general practice, with prospective recording of subsequent cancer. Fam Pract. 2013;30:153-60.

16. Neal RD, Allgar VL, Ali N, Leese B, Heywood P, Proctor G, et al. Stage, survival and delays in lung, colorectal, prostate and ovarian cancer: comparison between diagnostic routes. Br J Gen Pract. 2007;57:212-9.

17. Potter S, Govindarajulu S, Shere M, Braddon F, Curran G, Greenwood R, et al. Referral patterns, cancer diagnoses, and waiting times after introduction of two week wait rule for breast cancer: prospective cohort study. BMJ. 2007;335:288.

18. Jensen $H$, Torring ML, Olesen F, Overgaard J, Vedsted P. Cancer suspicion in general practice, urgent referral and time to diagnosis: a population-based GP survey and registry study. BMC Cancer. 2014;14:636. 
19. Jensen $H$, Torring ML, Larsen MB, Vedsted P. Existing data sources for clinical epidemiology: Danish Cancer in Primary Care (CaP) cohort. Clin Epidemiol. 2014;6:237-46.

20. Rothmann K, Greenland S, Lash TL. Modern Epidemiology. 3rd ed. Philadelphia: Lippencott Williams \& Wilkins; 2008.

21. Ferlay J, Shin H.R., Bray F, Forman D, Mathers C, Parkin DM. GLOBOCAN 2008, Cancer Incidence and Mortality Worldwide: IARC CancerBase No. 10. Version 3.0. 2012. Lyon: International Agency for Research on Cancer.

22. Gjerstorff ML. The Danish Cancer Registry. Scand J Public Health. 2011;39:42-5.

23. Danish National Board of Health: Det moderniserede Cancerregister - metode og kvalitet [the Modernised Cancer Registry - methods and quality]. Copenhagen S: Danish National Board of Health; 2009. http://www.ssi.dk/ /media/Indhold/ DK\%20-\%20dansk/Sundhedsdata\%20og\%20it/NSF/Registre/Cancerregisteret/ Det $\% 20$ moderniserede\%20Cancerregister\%20\%20metode\%20og\%20kvalitet.ashx] (Accessed 2-2-2013)

24. Pedersen CB. The Danish Civil Registration System. Scand J Public Health 2011;39:22-5.

25. Quan H, Li B, Couris CM, Fushimi K, Graham P, Hider P, et al. Updating and validating the Charlson comorbidity index and score for risk adjustment in hospital discharge abstracts using data from 6 countries. Am J Epidemiol. 2011:173:676-82.

26. UNESCO. International Standard Classification of Education ISCED 2011 Quebec, Canada: Montreal; 2012.

27. Ferlay J, Steliarova-Foucher E, Lortet-Tieulent J, Rosso S, Coebergh JW Comber $\mathrm{H}$, et al. Cancer incidence and mortality patterns in Europe: estimates for 40 countries in 2012. Eur J Cancer. 2013:49:1374-403.

28. Miranda A. QCOUNT: Stata program to fit quantile regression models for count data. 2006. Boston College Department of Economics.

29. Hao N. Quantile regression. Thousand Oaks, Calif: Sage Publications; 2007.

30. Machado JA, Santos Silva JMC. Quantiles for Counts. J Am Stat Assoc. 2005;100:1225-37.

31. Torring ML, Frydenberg M, Hamilton W, Hansen RP, Lautrup MD, Vedsted P. Diagnostic interval and mortality in colorectal cancer: U-shaped association demonstrated for three different datasets. J Clin Epidemiol. 2012;65:669-78,

32. van Harten MC, Hoebers FJ, Kross KW, van Werkhoven ED, van den Brekel MW, van Dijk BA. Determinants of treatment waiting times for head and neck cancer in the Netherlands and their relation to survival. Oral Oncol. 2014:51:272-78.

33. Torring ML, Frydenberg M, Hansen RP, Olesen F, Hamilton W, Vedsted P. Time to diagnosis and mortality in colorectal cancer: a cohort study in primary care. Br J Cancer. 2011;104:934-40.

34. Neal RD, Tharmanathan P, France B, Din NU, Cotton S, Fallon-Ferguson J, et al. Is increased time to diagnosis and treatment in symptomatic cancer associated with poorer outcomes? Systematic review. Br J Cancer. 2015;112 Suppl 1:S92-107.

35. Jensen $\mathrm{KH}$, Maina PJ. Cancer pathways are associated with improved long-term survival. Dan Med J. 2015:61:A5000.

36. Storm HH, Kejs AM, Engholm G. Improved survival of Danish cancer patients 2007-2009 compared with earlier periods. Dan Med Bull. 2011;58:A4346.

37. Deleuran T, Thomsen RW, Norgaard M, Jacobsen JB, Rasmussen TR, Sogaard M. Comorbidity and survival of Danish lung cancer patients from 2000-2011: a population-based cohort study. Clin Epidemiol. 2013;5:31-8.

38. Danish National Board of Health: National Cancer Plan II • Denmark National Board of Health recommendations for improving cancer healthcare services. Copenhagen: The National Board of Health; 2005.

39. Jones $R$, Rubin $G$, Hungin $P$. Is the two week rule for cancer referrals working? BMJ. 2001;322:1555-6.

40. O'Dowd A. GPs should be freed up to make more endoscopy referrals, cancer charity says. BMJ. 2014;348:g3602.

41. Larsen MB, Jensen $H$, Hansen RP, Olesen F, Vedsted P. Identification of patients with incident cancers using administrative registry data. Dan Med J. 2014;6:A4777.

42. Hansen RP: Delay in the diagnosis of cancer [PhD thesis]. Aarhus: Faculty of Health Sciences, University of Aarhus; 2008.

\section{Submit your next manuscript to BioMed Central and take full advantage of:}

- Convenient online submission

- Thorough peer review

- No space constraints or color figure charges

- Immediate publication on acceptance

- Inclusion in PubMed, CAS, Scopus and Google Scholar

- Research which is freely available for redistribution

Submit your manuscript at www.biomedcentral.com/submit 